\title{
Phagocytosis of Aged Human Neutrophils by Macrophages Is Mediated by a Novel "Charge-Sensitive" Recognition Mechanism
}

\author{
John S. Savill, Peter M. Henson, ${ }^{\star}$ and Christopher Haslett \\ Department of Medicine, Royal Postgraduate Medical School, Hammersmith Hospital, London W12 OHS, United Kingdom; and \\ *National Jewish Center for Immunology and Respiratory Medicine, Denver, Colorado 80206
}

\begin{abstract}
The removal of neutrophils and their histotoxic contents from the inflamed site is a prerequisite for resolution of tissue injury, and a point at which factors critical to the pathogenesis of chronic inflammation may act. Engulfment of intact, senescent neutrophils by macrophages represents an important neutrophil disposal process. In this study the mechanism by which human monocyte-derived macrophages $(M \phi)$ recognized and ingested human neutrophils that had been aged in culture was studied using an in vitro phagocytic assay. Inhibition of $\mathbf{M \phi}$ receptors for Ig Fc and the opsonic complement fragments C3b and iC3b with MAbs to M $\phi$ FcR, CR1, CR3, and CR4 had no effect on recognition, and the pattern of inhibition observed when polyanions were included in the medium at $1 \mathrm{mg} / \mathrm{ml}$ was different from that reported for the $M \phi$ receptor for protein advanced glycosylation end products (AGE), indicating a recognition mechanism different from those proposed for $\mathrm{M} \phi$ phagocytosis of senescent erythrocytes. Furthermore, although aging neutrophils undergo programmed cell death (or apoptosis), which is directly related to recognition by $M \phi$, the pattern of inhibition observed with monosaccharides was different from that reported to inhibit the binding of apoptotic mouse thymocytes to isologous $M \phi$. By contrast, evidence was obtained for a novel recognition mechanism inhibitable by cationic sugars and amino acids in a charge-dependent fashion, and directly modulated by $\mathrm{pH}$ but not affected by inhibitors of the mannose-6-phosphate, sheep erythrocyte, mannosyl-fucosyl, asialoglycoprotein, and scavenger receptors of the macrophage. These observations suggest that hydrogen ions and charged molecules may modulate $M \phi$ uptake of senescent neutrophils at inflamed sites, and that recognition itself may involve charged structures on the cells.
\end{abstract}

\section{Introduction}

Macrophage phagocytosis of apparently intact neutrophils has been described at inflamed sites since the time of Metchnikoff (1), although the changes in the neutrophil responsible for recognition as nonself have remained obscure until recently $(2$, 3). Human monocyte-derived macrophages $(\mathrm{M} \phi),{ }^{1}$ during the

Address correspondence to Dr. John S. Savill, Respiratory Division, Department of Medicine, Royal Postgraduate Medical School, Hammersmith Hospital, Du Cane Rd., London W12 OHS, UK.

Received for publication 13 October 1988 and in revised form 17 July 1989.

1. Abbreviations used in this paper: $\mathrm{AGE}$, advanced glycosylation end products; AGE-R, M $\phi$ receptor for AGE; EIgG, ox red cells opsonized

J. Clin. Invest.

(c) The American Society for Clinical Investigation, Inc.

$0021-9738 / 89 / 11 / 1518 / 10 \$ 2.00$

Volume 84, November 1989, 1518-1527 process of maturation from monocytes in vitro, gain the capacity to recognize and ingest human neutrophils that have been aged in culture for $\sim 24 \mathrm{~h}$ after purification from the blood of normal donors (2). The phagocytic nature of the interaction was proven by electron microscopy with postfixation incubation with cationized ferritin; phagosomes containing aged neutrophils did not admit ferritin (2). Freshly isolated neutrophils were not ingested, but needed to undergo a time-dependent aging process before recognition and phagocytosis by macrophages occurred. Subsequently, it was shown that neutrophils isolated from normal blood or inflamed sites undergo morphological and biochemical changes typical of programmed cell death or apoptosis during aging in vitro (3-5), and that this process was directly related to recognition of the aging cell by M $\phi$. Furthermore, there was evidence that neutrophil apoptosis leading to recognition of the intact cell by macrophages occurred at inflamed sites in vivo, representing a mechanism for disposal of neutrophils that may serve to limit the degree of tissue injury (3).

However, the cell surface interactions involved in $\mathrm{M} \phi$ recognition of aged neutrophils have not been examined hitherto, and are the subject of this study. Macrophage receptors for opsonins (Ig Fc and the complement fragments $\mathrm{C} 3 \mathrm{~b}$ and $\mathrm{iC} 3 \mathrm{~b}$ ) and for advanced glycosylation end products of proteins (AGE) have been implicated in $\mathrm{M} \phi$ recognition of the senescent erythrocyte (6-10). A possible role for these mechanisms in aged neutrophil recognition was sought in this study, although an opsonic mechanism appears unlikely to account for M $\phi$ recognition of aged neutrophils since, by contrast with senescent erythrocyte recognition, serum is not required (2), but it remains possible that cytophilic antibody persisting in culture, or $\mathbf{M} \phi$-derived complement fragments could mediate phagocytosis by such a mechanism. Furthermore, the time course of glycosylation of cell-surface proteins $(9,10)$ would appear to be too slow to account for the recognition of neutrophils that have been aged for $24 \mathrm{~h}$ or less. However, since it has been proposed that the binding of apoptotic mouse thymocytes by isologous $\mathbf{M} \phi$ occurs by a sugar-lectin mechanism involving a macrophage lectin inhibitable by monosaccharides such as $N$-acetylglucosamine (11), these studies also sought a role for such a mechanism in $\mathbf{M} \phi$ recognition of aged neutrophils, which undergo apoptosis (3).

In the present study evidence was obtained that neither $\mathbf{M} \phi$ receptors for opsonins ( $\mathrm{Fc}, \mathrm{C} 3 \mathrm{~b}$, and $\mathrm{iC} 3 \mathrm{~b}$ ), nor those for $\mathrm{AGE}$, participated in the recognition of aged neutrophils, and no evidence in support of a role for an $N$-acetylglucosamine-specific lectin was found. Indeed, inhibitors of a number of other M $\phi$ receptors with properties that suggested they might have played a role in recognition of aged neutrophils failed to influence the interaction. However, recognition was inhibited by

with rabbit IgG; MPO, myeloperoxidase; $M \phi$, monocyte-derived macrophages. 
cationic monosaccharides and amino acids in a $\mathrm{pH}$ - and charge-dependent fashion, and was directly modulated by $\mathrm{pH}$, suggesting that the $\mathbf{M} \phi$ recognition of aged neutrophils occurs by a novel charge-sensitive mechanism.

\section{Methods}

Materials. All chemicals were obtained from Sigma Chemical Co., St. Louis, MO, unless otherwise indicated; culture media were from Gibco Laboratories, Grand Island, NY; and sterile tissue culture plasticware was purchased from Falcon Plastics, Cockeysville, MD.

Cells. Neutrophils ( $>98 \%$ pure) were isolated from fresh, citrated normal human blood by dextran sedimentation and plasma-Percoll (Pharmacia Fine Chemicals, Piscataway, NJ) density gradient centrifugation and aged in culture for $24 \mathrm{~h}$ at $37^{\circ} \mathrm{C}$ as previously described (3, 12). In some experiments inflammatory neutrophils were purified from rheumatoid synovial fluid as described (3). Human $\mathbf{M} \phi$ were prepared by standard methods from adherent PBMC by culture for $7 \mathrm{~d}$ in 24-well plates as described (3). Opsonized ox erythrocytes (EIgG) were prepared by standard methods using polyclonal rabbit anti-ox erythrocyte IgG (a gift of Dr. Grennan). Ox red cells were washed three times in PBS, incubated at room temperature for $30 \mathrm{~min}$ with antibody at $\sim 10 \mu \mathrm{g} \mathrm{ml}$, and then washed three more times before resuspension in the appropriate medium (see below).

Interaction assay. This phagocytic assay, based on that used in earlier work (2), was as previously described (3). Briefly, aged neutrophils were washed in HBSS and then suspended in HBSS at 5 $\times 10^{6} / \mathrm{ml}$. $1 \mathrm{ml}$ of suspension was then added to each washed well of mature $\mathrm{M} \phi$ and interaction allowed to occur at $37^{\circ} \mathrm{C} / 5 \% \mathrm{CO}_{2}$ and $\mathrm{pH}$ 7.4 for $30 \mathrm{~min}$, during which the neutrophils settled into a carpet in close relation to the $\mathbf{M} \phi$ monolayer. The interaction was terminated by vigorous washing of the wells with cold $\left(4^{\circ} \mathrm{C}\right) 0.9 \%$ saline, and $\mathrm{M} \phi$ were fixed in $2 \%$ glutaraldehyde in PBS. M $\phi$ interacting with aged neutrophils were identified by staining for myeloperoxidase (MPO) using hydrogen peroxide and dimethoxybenzidine (o-dianisidine $\mathrm{HCl})$ as a substrate (see references 2 and 13 for details). After $7 \mathrm{~d}$ maturation the M $\phi$ themselves were $100 \%$ MPO negative. Aged neutrophils were $100 \%$ MPO positive. The magnitude of the interaction was quantified by inspection of five randomly selected microscope fields in each well so that at least $500 \mathrm{M} \phi$ were examined. Macrophages were scored as positive if they contained one or more masses of MPO-positive material which usually had the appearance of intact neutrophils. The vigorous washing used dislodged neutrophils that had not been ingested (Fig. $4 A$ and $B$ ) but the occasional aged neutrophil appeared to project beyond the outline of the $\mathbf{M} \phi$. The earlier ultrastructural studies (2) suggested that even these had been engulfed by the macrophages (presumably they may be surrounded by a thin cytoplasmic covering not visible by light microscopy) and that the neutrophils were rapidly ingested and did not remain in an attached state. However, to minimize any potential overestimate of phagocytosis, neutrophils that appeared to project by more than half their diameter beyond the outline of the macrophage were not counted. Results were recorded for each well as the percentage of $M \phi$ ingesting aged PMN. Previous work $(2,3)$ has shown that this simple assay correlates well with assessment of interaction by radiolabeling neutrophils and measuring radioactivity in the wells after interaction.

M $\phi$ uptake of opsonized erythrocytes was assayed by similar methods, $1 \mathrm{ml}$ of a suspension at $5 \times 10^{6} / \mathrm{ml}$ in HBSS being added to each well before an identical incubation. After washing with cold $\left(4^{\circ} \mathrm{C}\right)$ $0.9 \%$ saline, noningested EIgG were removed by lysis with cold $0.2 \%$ saline, followed by an equal volume of $1.6 \%$ saline before washing, fixing, staining for peroxidative activity, and quantitation of the interaction by the same methods as for neutrophils. In all experiments $>95 \%$ of $\mathrm{M} \phi$ recognized and ingested EIgG.

Monosaccharide and amino acid effects. $\mathrm{M} \phi$ recognition of aged neutrophils and EIgG were assessed after inclusion of these molecules at the desired concentration and $\mathrm{pH}$ in the interaction medium. Mono- saccharides and amino acids (all in the $\mathrm{D}[+]$ stereroisomeric form unless stated; Sigma Chemical Co.) were dissolved in HBSS at the desired concentration. If necessary, the $\mathrm{pH}$ was adjusted by addition of small quantities of concentrated sodium hydroxide or hydrochloric acid (the small differences in osmolality, ionic strength, and sodium and chloride concentrations induced by this procedure had no independent effect on recognition as assessed by use of HBSS with the appropriate concentration of sodium chloride added; data not shown). After being washed in HBSS at the appropriate $\mathrm{pH}$ and added to the $\mathbf{M} \phi$, aged neutrophils were suspended at the standard concentration in the appropriately treated HBSS. According to cell availability, between two and five replicates of each treatment were included in each experiment; results were expressed as mean \pm SEM percentage of control (the mean percentage of $\mathrm{M} \phi$ ingesting aged neutrophils in wells simultaneously assayed under standard conditions, as above). In some experiments aged PMN or M $\phi$ were preincubated with HBSS containing sugars or amino acids; the cells were incubated in such media at $4^{\circ} \mathrm{C}$ for $30 \mathrm{~min}$ and then washed before interaction under standard conditions.

Polyanion effects. These were assayed by the same methods, polyanions being included in the interaction medium at $\mathrm{pH}$ 7.4. These included heparin, fucoidin, dextran sulfate, and $\alpha$-mannosidase (from Jack beans; Sigma Chemical Co.). Other inhibitors used were also from Sigma Chemical Co. and included ganglioside GDla, neuraminyl-lactose, mannan, and ribonuclease $\mathrm{B}$.

Effects of mouse MAb inhibition of $M \phi$ receptors for $F c, C 3 b$, and $i C 3 b$. These were assessed by adaptations of the interaction assay described above, designed to reduce the possibility that $\mathbf{M} \phi$ might internalize and inactivate blocking antibody. Washed $\mathrm{M} \phi$ were preincubated with antibody (or medium alone) at $4^{\circ} \mathrm{C}$ for $15 \mathrm{~min}$ in $300 \mu \mathrm{l}$ of medium. Aged neutrophils $\left(5 \times 10^{6}\right)$ were added at the end of this time in $100 \mu \mathrm{l}$ of medium, and interaction was allowed to occur at $37^{\circ} \mathrm{C}$ in the presence of antibody excess for a further $15 \mathrm{~min}$ before washing, fixing, staining, and counting as above. Blockade was verified by the simultaneous demonstration in other wells on the same plate of $\mathbf{M} \phi$, that identical procedures inhibited $\mathrm{M} \phi$ recognition of $5 \times 10^{6}$ xenogenic red cells coated by standard methods (14-16) with the appropriate ligand: EIgG for the FcR and sheep red cells coated with $\mathrm{C} 3 \mathrm{~b}$ (EC3b) or iC3b (EiC3b; these and EC3b were the gift of Dr. Gordon D. Ross). Preliminary experiments (Savill, J. S., G. D. Ross, and C. Haslett, unpublished data) demonstrated that to inhibit $\mathrm{M} \phi \mathrm{FcR}$ function by over $90 \%$, i.e. reduce uptake of EIgG to $<10 \%$ of control, it was necessary to use antibodies directed against FcRI, FcRII, and FcRIII simultaneously (i.e., antibodies 10.1 , IV.3 and 3G8, kindly provided by Dr. Gordon Ross, Dr. Nancy Hogg, and their colleagues), each at 25 $\mu \mathrm{g}$ per well. Inhibition of EC3b binding by $\mathbf{M} \phi$ down to $\sim 10 \%$ of control (binding being assessed by counting the proportion of $M \phi$ still forming rosettes with $\mathrm{E} 3 \mathrm{Cb}$ after washing in cold saline without a lysis step) was achieved using $150 \mu \mathrm{g}$ per well of a Fab'2 preparation of a polyclonal anti-CR1 antibody (a gift of Dr. Gordon Ross). To inhibit $\mathrm{M} \phi$ binding of EiC3b to a similar degree, three antibodies, MN41, Leu 15, and OKM1, directed against three distinct epitopes on the CR3 molecule $(14,15)$ were necessary, each at $25 \mu \mathrm{g}$ per well. EiC $3 \mathrm{~b}$ binding diminished by these antibodies could be further reduced to $<5 \%$ of control, evidence of inhibition of CR4-mediated iC $3 \mathrm{~b}$ binding $(15,16)$, by the addition, each at $25 \mu \mathrm{g} /$ well, of three antibodies to p150,95 (LeuM5, 3.9, and L-29, gifts of Dr. Gordon Ross). Subsequently, assessment of aged neutrophil recognition with simultaneous verification of CR4 inhibition was not practicable because of shortage of antibody.

\section{Results}

Recognition of aged neutrophils by macrophages. In a large number of experiments the proportion of the $\mathbf{M} \phi$ recognizing aged neutrophils under standard conditions was $46.1 \pm 2.3 \%$ (mean \pm SEM, $n=159$ ), but this varied from experiment to 
experiment, with a range of $13-86 \%$. However, in each experiment between two and five replicates were included, and when the proportion of $\mathbf{M} \phi$ recognizing aged neutrophils in a given experiment was expressed as a percentage of the mean of the replicates in that experiment, $95 \%$ of observations fell within $9.6 \%$ of the mean (Fig. 1). Therefore, although there was variation in recognition of aged neutrophils between experiments, the assay of recognition was highly reproducible within an individual experiment, and inhibitory effects on recognition were compared between experiments by expressing recognition within each experiment as percent of the mean of control wells (i.e., wells on the same plate of $\mathbf{M} \phi$ in which recognition was assayed under standard, control conditions). The percentage of macrophages recognizing aged $P M N$ in each series of experiments is given as mean $\pm \mathrm{SE}$ in the legend of each figure or table.

No effect of inhibition of $M \phi F c$, complement, and $A G E$ receptors. When $\mathrm{M} \phi$ receptors for $\mathrm{IgG} \mathrm{Fc}, \mathrm{C} 3 \mathrm{~b}$, and $\mathrm{iC} 3 \mathrm{~b}$ were inhibited by preincubation with mouse MAbs to FcR, CR1, CR3, and CR4 (see Methods), blockade being verified in the same experiment by the confirmation of reduced recognition of xenogenic red cells coated with the appropriate ligand, no effect on $\mathbf{M} \phi$ recognition of aged neutrophils was observed (Table I). The remote possibility that antibodies might be blocking recognition by one class of receptor, but opsonizing the neutrophils for another to a compensating degree (e.g., anti-CR3 antibodies might bind to CR3 on the aged neutrophils and opsonize them for the M $\phi$ FcR) was excluded by: (a) simultaneous blockade within the same well of $M \phi F c R$ and CR3 or CR4 (Table I); (b) the use of Fab'2 antibody fragments (i.e., anti-CR 1); and (c) failure of any of the antibody combinations used to induce ingestion of freshly isolated neutrophils assayed under identical conditions (data not shown).

M $\phi$ AGE receptors can be competitively inhibited by polyanions such as heparin and fucoidin $(9,10)$, but when these molecules were included in interactions at $1 \mathrm{mg} / \mathrm{ml}$, a concentration well in excess of that required to inhibit the AGE receptor $(9,10)$, fucoidin had no effect (Fig. 2). Although heparin inhibited phagocytosis of aged neutrophils by macrophages in a concentration-related manner, dextran sulfate (an inhibitor of the $\mathbf{M} \phi$ scavenger receptor) was inactive. Heparin did not affect M $\phi$ uptake of EIgG at the same concentrations (Fig. 2).

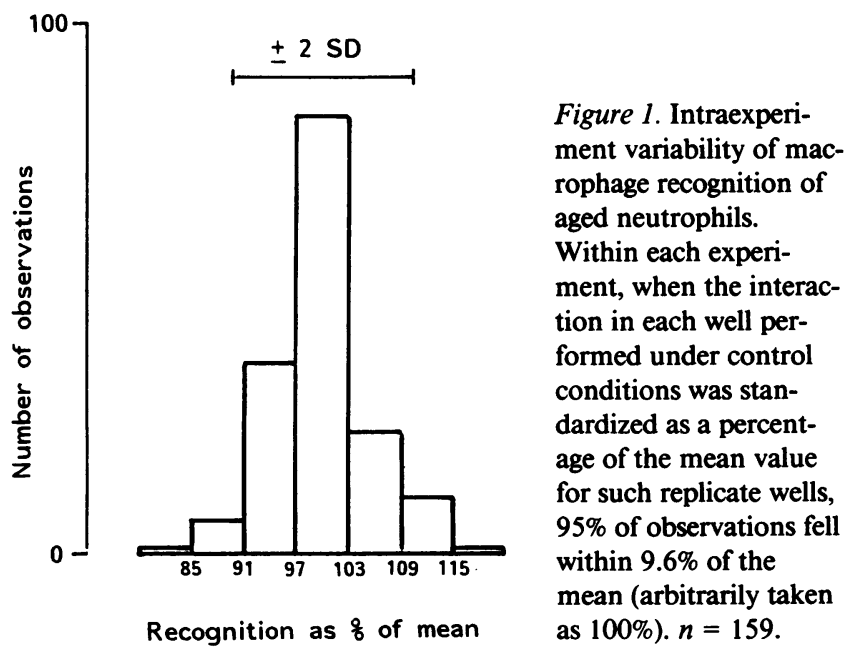

Table I. Effect of Mouse Antibody Inhibition of $M \phi F c$ and Complement Receptors on Recognition of Aged Neutrophils or Xenogenic Erythrocytes (E) Opsonized with the Appropriate Ligand

\begin{tabular}{lcl}
\hline $\begin{array}{c}\text { Receptor } \\
\text { inhibited }\end{array}$ & $\begin{array}{c}\text { Recognition of aged } \\
\text { neutrophils }\end{array}$ & Recognition of opsonized E \\
\hline & \multicolumn{2}{c}{ mean \% of control SEM } \\
FcR & $99.4 \pm 1.9(n=11)$ & EIgG; $6.1 \pm 1.5(n=5)$ \\
CR1 & $99.8 \pm 5.4(n=5)$ & EC3b; $12.4 \pm 2.0(n=4)$ \\
CR3 & $97.2 \pm 3.9(n=10)$ & EiC3b; 10.1 $13.2(n=5)$ \\
CR4 & $104.6 \pm 4.3(n=8)$ & ND* \\
CR3 + FcR & $93.8 \pm 5.4(n=6)$ & ND \\
CR4 + FcR & $102.5 \pm 3.1(n=4)$ & ND \\
& & \\
\hline
\end{tabular}

* See Methods. There was insufficient antibody to confirm inhibition of CR4-mediated EiC3b binding in these experiments, but this was done in preliminary experiments (not shown). As explained in Methods, $\mathrm{M} \phi$ interaction with $\mathrm{EC} 3 \mathrm{~b}$ and $\mathrm{EiC} 3 \mathrm{~b}$ was assayed by assessment of the proportion of $\mathrm{M} \phi$ binding coated erythrocytes after washing in cold saline; for recognition of EIgG, phagocytosis was confirmed by hypotonic lysis of noningested cells. In this series of experiments $46.1 \pm 3.3 \%$ of $\mathrm{M} \phi$ recognized aged $\mathrm{PMN}$ under control conditions; $95 \%$ of observations fell within $10.4 \%$ of the mean of control (100\%).

Inhibition by amino sugars but not by $N$-acetylglucosamine. When a range of monosaccharides were included in the

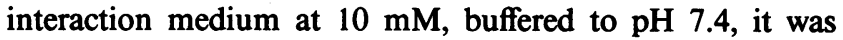
observed that the amino sugars glucosamine, galactosamine, and mannosamine inhibited $\mathbf{M} \phi$ uptake of aged neutrophils (Figs. 3 and 4). The lack of effect on phagocytosis of ox erythrocytes opsonized with rabbit IgG (EIgG) provided evidence against inhibition being due to a nonspecific toxic effect of amino sugars on the M $\phi$. Furthermore, these observations did not appear to be due to some toxic effect of amino sugars on the neutrophils, since there was no change in the percentage of neutrophils excluding trypan blue when aged neutrophils were incubated with amino sugars under conditions identical to those resulting in inhibition of recognition (Fig. 3). The effect of amino sugars on aged neutrophil recognition was concentration related (Fig. 5; data for glucosamine shown). Despite variation in the proportion of macrophages recognizing aged neutrophils under control conditions, as reported above, the

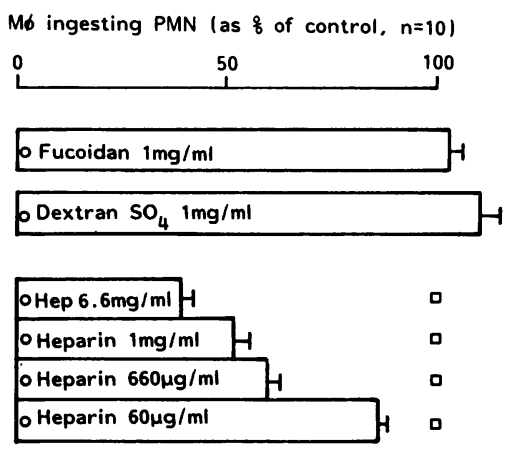

Figure 2. Effect of polyanions on recognition of aged neutrophils and EIgG by $M \phi$. At 1 $\mathrm{mg} / \mathrm{ml}$ in the interaction medium fucoidin and dextran sulfate had no effect on uptake of aged neutrophils into $\mathbf{M} \phi$, whereas heparin was inhibitory in a concentration-dependent manner (open bars). No effect was seen on uptake of EIgG (open squares). None of the treatments affected aged neutrophil viability assessed by exclusion of trypan blue (open circles). In these experiments $33.1 \pm 1.7 \%$ of $\mathrm{M} \phi$ recognized aged PMN under control conditions. 
Prnportion Mø ingesting PMN (as: of control)

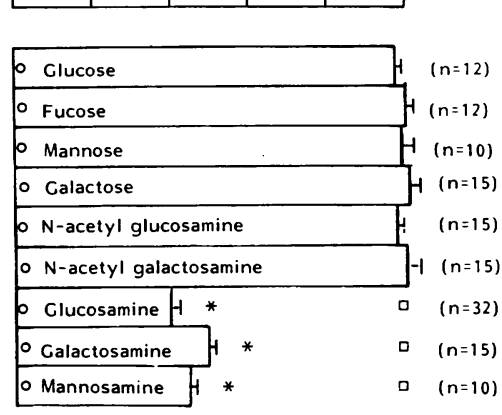

$\begin{array}{llllll}0 & 20 & 40 & 60 & 80 & 100\end{array}$

Figure 3. Effect of 10 mM monosaccharides on $\mathbf{M} \phi$ recognition of aged neutrophils at $\mathrm{pH}$ 7.4. Amino sugars included in the interaction medium, but not other sugars, inhibited $\mathbf{M} \phi$ recognition of aged neutrophils (open bars) but not recognition of EIgG (open squares). Neutrophil viability assessed by trypan blue exclusion (open circles)

was not affected by the conditions used in these experiments. In these experiments $49.5 \pm 2.5 \%$ of $\mathrm{M} \phi$ recognized aged neutrophils under control conditions. ${ }^{*} P<0.001$.

degree of inhibition associated with a given concentration of glucosamine did not appear to be related to the magnitude of the control interaction in an extended range of experiments where under control conditions the percentage of macrophages recognizing aged PMN varied from 12.3 to $89.9 \%, n$ $=58$. It was found that in experiments where between 10.0 and $29.9 \%$ of $\mathrm{M} \phi$ recognized aged PMN under control conditions, $10 \mathrm{mM}$ glucosamine in the interaction reduced recognition to $41.3 \pm 9.3 \%$ of control (\%C, mean $\pm \mathrm{SD}, n=22)$; with control interactions between 30.0 and $49.9 \%$ there was inhibition to $36.0 \pm 10.4 \% \mathrm{C}, n=13$; between 50.0 and $69.9 \%$ to $40.3 \pm 9.9 \% \mathrm{C}, n=1$; and between 70.0 and $89.9 \%$ to $34.4 \pm 8.8 \% \mathrm{C}, n=13$. At the maximum concentration used ( 50 $\mathrm{mM}$ ), none of the sugars tested, other than the amino sugars, induced inhibition (data not shown). In particular, no effect was observed with $N$-acetylglucosamine, $N$-acetylgalactosamine, or galactose, the sugars reported to inhibit the binding of apoptotic mouse thymocytes to isologous $\mathbf{M} \phi$ (11), even when these sugars were preincubated with $\mathrm{M} \phi$ at 10 and 50 $\mathrm{mM}$ for $30 \mathrm{~min}$ at $4^{\circ} \mathrm{C}$ (data not shown), a maneuver that had inhibited apoptotic thymocyte binding (11).

When $\mathrm{M} \phi$ or aged neutrophils (at $5 \times 10^{6} / \mathrm{ml}$ ) were preincubated with $10 \mathrm{mM}$ monosaccharides for $30 \mathrm{~min}$ at $4^{\circ} \mathrm{C}$, washed, and then interacted under standard conditions, it was found that preincubation of aged neutrophils (but not $\mathbf{M} \phi$ ) with amino sugars (but not glucose) inhibited subsequent recognition (Fig. 6). Thus inhibition of $\mathrm{M} \phi$ recognition of aged neutrophils by sugars appeared to be specific, concentration related, and related in large part to an effect at the neutrophil surface.

Inhibition by cationic amino acids. It was noted that neither the parent sugars nor the $N$-acetylated derivatives of the amino sugars inhibited recognition of aged neutrophils by $\mathbf{M} \phi$ (Fig. 3). A salient difference between these sugars and the relevant amino sugar is that the free amino group allows the amino sugars to act as weak bases, such that at $\mathrm{pH} 7.4$ the majority of glucosamine molecules, for example, are cationically charged. Thus it appeared possible that the inhibitory effect of amino sugars might relate to their charge, and to investigate this possibility further amino acids of neutral, anionic, and cationic charge were included in the interaction medium at $10 \mathrm{mM}$ buffered to $\mathrm{pH}$ 7.4. The neutrally charged D-glutamine and L-glutamine had no effect, nor did the an- ionic L-glutamic acid, but L-lysine and L-arginine, which have cationically charged $\mathrm{R}$ groups at $\mathrm{pH} 7.4$, inhibited $\mathrm{M} \phi$ recognition of aged neutrophils but not EIgG (Fig. 7).

Effect of $p H$ on $M \phi$ recognition of aged neutrophils. Since the inhibitory effect of amino sugars and basic amino acids may depend on their capacity to bear protons, the effect of hydrogen ion concentration itself on macrophage recognition of aged neutrophils was investigated. Within the range $\mathrm{pH}$ 6.5-8.5 there was no effect on the ability of aged neutrophils to exclude trypan blue nor on $\mathrm{M} \phi$ recognition of EIgG (Fig. 5), although out of this range these parameters were adversely affected (data not shown). By contrast, $\mathbf{M} \phi$ recognition of aged neutrophils was significantly modulated by $\mathrm{pH}$; at $\mathrm{pH} 6.5 \mathrm{rec}-$ ognition was inhibited, while at $\mathrm{pH} 8.5$ it was enhanced relative to pH 7.5 (Fig. 8).

Dependency of amino sugar and amino acid inhibition on charge. It was possible to investigate whether the inhibitory effect of a given amino sugar or amino acid depended on bearing a cationic charge, as suggested by the preceding data, by varying the $\mathrm{pH}$ at which the interaction was performed and calculating the proportion of molecules bearing protons from the $\mathrm{pK}$ and the Henderson-Hasselbach equation (17). The degree of inhibition observed with $10 \mathrm{mM}$ glucosamine relative to control at the same $\mathrm{pH}$ was correlated with this proportion (Fig. 9). The reduction of inhibitory effect at $\mathrm{pH} 8.5$ appeared not to be related to the $\mathrm{pH}$ itself, since $10 \mathrm{mM}$ L-lysine inhibited at $\mathrm{pH} 8.5$ where this molecule is still predominantly cationic (Fig. 9). Indeed, $10 \mathrm{mM}$ histidine, which is predominantly neutral at $\mathrm{pH} 7.5$ but $33 \%$ cationic at $\mathrm{pH} 6.5$, only exerted an inhibitory effect on $\mathbf{M} \phi$ recognition of aged neutrophils at the $\mathrm{pH}$ at which it bore a cationic charge (Fig. 9). Thus, at least for glucosamine and histidine, the inhibitory effect of the molecule on macrophage recognition was directly related to the cationic charge on the molecule, be it amino sugar or amino acid. It did not appear likely that the lack of abrogation of the inhibitory effect of L-lysine at pH 8.5 reflected inhibition by a mechanism different from glucosamine since, just as had been shown for glucosamine, preincubation of aged neutrophils for $30 \mathrm{~min}$ at $4^{\circ} \mathrm{C}$ and $\mathrm{pH} 7.5$ with $10 \mathrm{mM}$ L-lysine, followed by washing, inhibited subsequent recognition of these cells (to $59.5 \pm 2.1 \% \mathrm{C}, n=6$ ). Preincubation with $\mathrm{M} \phi$ had no effect.

Relationship of aged neutrophil recognition mechanism to known macrophage receptors. There were grounds to examine the effects on the interaction of inhibitors of a number of well-characterized macrophage receptors (in addition to those for $\mathrm{Fc}$, iC $3 \mathrm{~b}, \mathrm{C} 3 \mathrm{~b}$, and AGE). First, receptors expressed by mature macrophages and known to have negatively charged ligands (i.e., the mannose-6-phosphate, sheep erythrocyte, and scavenger receptors) were examined since the polyanion heparin had inhibited the interaction. No effect was seen with concentrations of inhibitors such as mannose-6-phosphate accepted as suprainhibitory by previous investigators (see Table II for data and references). Second, human $\mathbf{M} \phi$ receptors with sugar specificities similar to that of the lectin-like structure proposed to mediate binding of isologous apoptotic thymocytes (11; inhibited by $N$-acetylglucosamine, $N$-acetylgalactosamine, and galactose) were examined. Inhibitors of the mannosyl-fucosyl receptor (which may recognize residues bearing $\mathrm{N}$-acetylglucosamine; 18) and asialoglycoprotein/galactosyl receptor had no effect on $\mathbf{M} \phi$ recognition of aged neutrophils (Table II). 

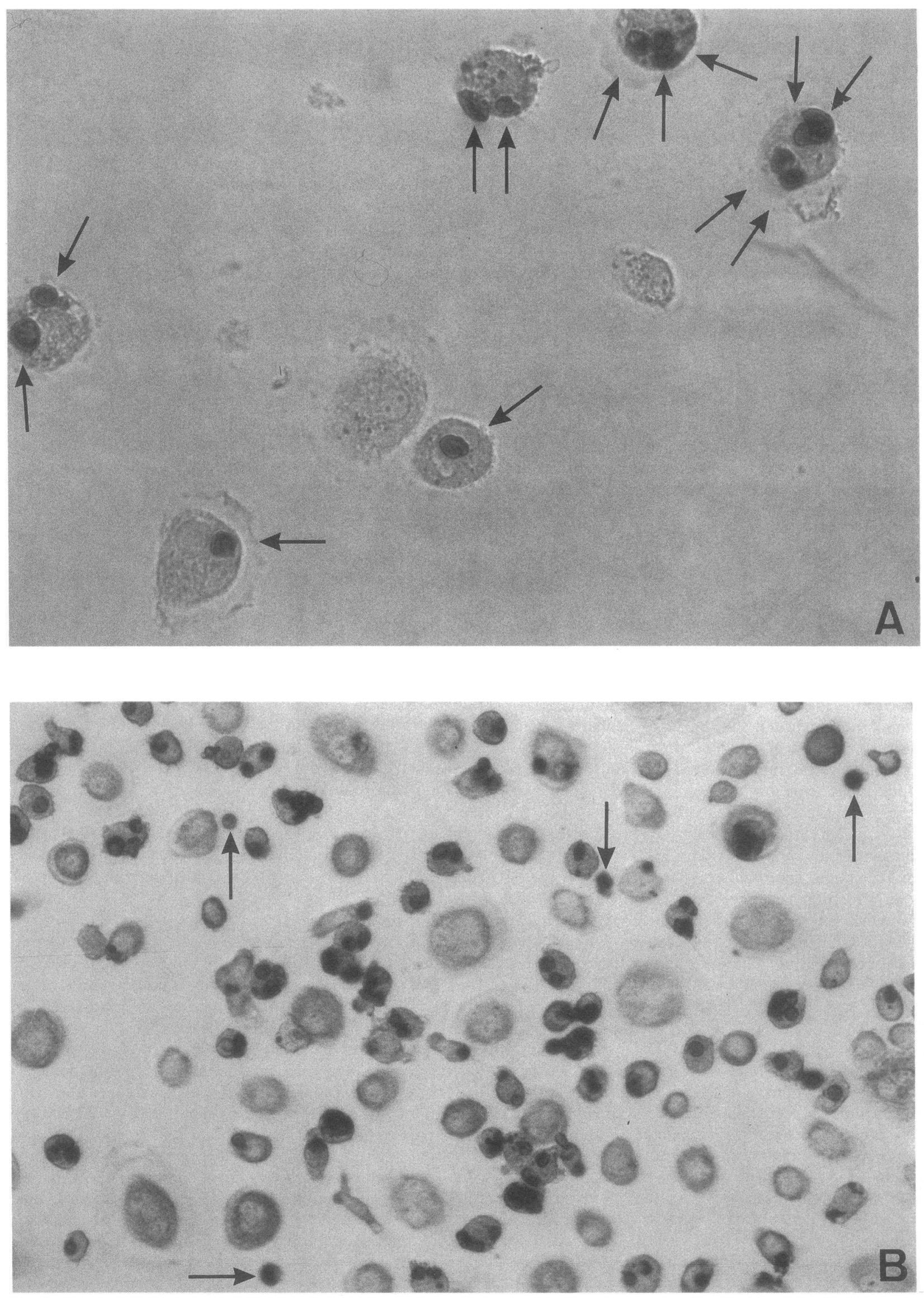


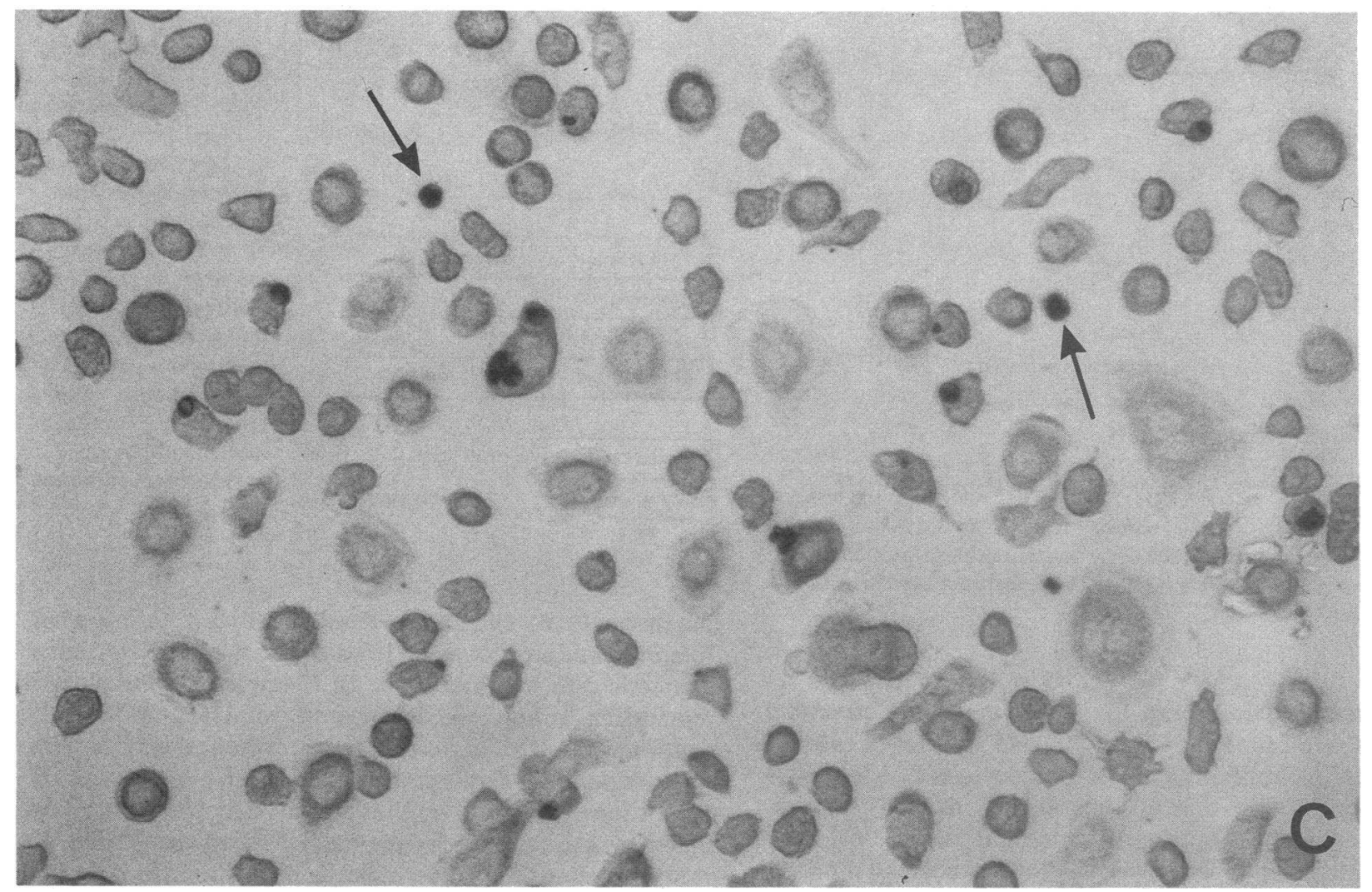

Figure 4. Appearances of control and glucosamine-inhibited assays of macrophage recognition of aged neutrophils. A, A high power view $(\times 1,000)$ of an interaction performed under control conditions is shown, a sparse area of the macrophage culture being shown for clarity. Those macrophages that have recognized aged neutrophils contain one or more brown-staining, MPO-positive bodies (arrows), some of which are already in early stages of fragmentation and digestion within the macrophage. Two macrophages in the center of the field have not ingested aged neutrophils. $B$, A positive interaction assay is shown at the magnification at which the interaction is scored $(\times 250)$. In this well $59.1 \%$ of $\mathrm{M} \phi$ recognized aged neutrophils. The interacting macrophages are easily distinguished by their content of MPO-positive aged neutrophils. Four noninteracting aged neutrophils, some of which have spread on the culture surface, have not been removed by washing (arrows) but are easily distinguished from macrophages on the basis of size and pattern of MPO positivity. $C, 50 \mathrm{mM}$ glucosamine was present in the interaction solution; $11.1 \%$ of $\mathrm{M} \phi$ recognized aged neutrophils, $18.8 \%$ of control. Two noninteracting neutrophils are seen (arrows).

Macrophage recognition of human inflammatory neutrophils. The preceding data refer to neutrophils that had been isolated from the blood of healthy donors. Previous work showed that viable inflammatory neutrophils from the synovial fluid of patients with rheumatoid arthritis also became recognizable to $\mathbf{M} \phi$ in a time-dependent fashion (3). In common with aged neutrophils prepared from the circulating pool of normal donors, recognition of aged joint-derived neutrophils could also be inhibited in this study by $10 \mathrm{mM}$ glucosamine (to $37.1 \pm 2.4 \%$ of control, $n=9$ ) and $10 \mathrm{mM}$ L-lysine (47.6 \pm 6.3 of control, $n=9$ ), but not by the control sugars, glucose $(99.6 \pm 2.5 \%, n=9)$, and $N$-acetylglucosamine $(96.6 \pm 1.4 \%, n=9)$, or the control amino acid, glutamine $(96.0 \pm 2.3 \%, n=9)$, in the same experiments.

\section{Discussion}

In this study we report that phagocytosis of aging neutrophils in vitro by $\mathbf{M} \phi$ may be specifically modulated by charged molecules and $\mathrm{pH}$, but not by inhibitors of $\mathrm{M} \phi$ receptors for opsonic complement fragments, $\mathrm{FcR}$, or advanced glycosylation end products of proteins, three classes of receptor for which direct evidence exists to indicate a role in $\mathbf{M} \phi$ recognition of senescent erythrocytes (6-10). Furthermore, although aging neutrophils undergo changes typical of programmed cell death or apoptosis (3-5), no evidence was found in support of a role in aged neutrophil phagocytosis for the cell surface sugar-lectin interaction proposed for $\mathbf{M} \phi$ binding of apoptotic thymocytes (11). $\mathbf{M} \phi$ phagocytosis of apparently intact neutrophils at inflamed sites has been described since the time of Metchnikoff (1, 19-24), and seems likely to represent an important neutrophil disposal mechanism $(3,23,24)$ with the potential to limit inflammatory tissue injury by removing neutrophils without the inevitable release of contents with demonstrated histotoxic properties $(25,26)$. Failure of the recognition and uptake process may represent an event favoring persistence of inflammation and progression rather than resolution of tissue injury. Our data suggest that this cellular interaction depends upon a novel charge-sensitive recognition mechanism with properties that imply that the extracellular microenvironment of the inflamed site may influence removal of intact senescent neutrophils.

Macrophage phagocytosis of senescent erythrocytes, although enucleate, might have served as a model for the recognition of aged neutrophils. However, although $\mathbf{M} \phi$ receptors for $\mathrm{Fc}, \mathrm{C} 3 \mathrm{~b}$, and $\mathrm{iC} 3 \mathrm{~b}$ have been implicated in the recognition 


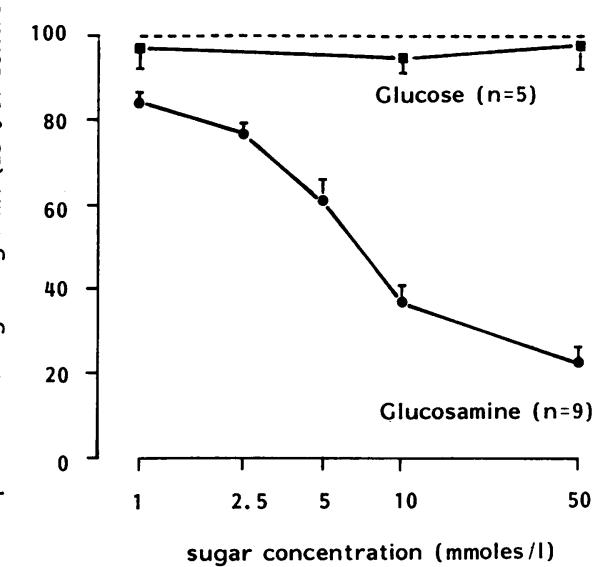

Figure 5. Concentration-related inhibition of aged neutrophil uptake by glucosamine. Glucose at the same concentrations was ineffective. In these experiments $55.0 \pm 8.8 \%$ of $M \phi$ recognized aged neutrophils under control conditions.

of senescent erythrocytes (6-8), inhibition of these structures with MAbs to M $\phi, F c R, C R 1, C R 3$, and CR4 failed to affect aged PMN recognition (and $M \phi$ phagocytosis of IgG-opsonized ox erythrocytes was not inhibited by the molecules inhibiting aged PMN uptake). Furthermore, despite evidence that the $\mathrm{M} \phi$ receptor for advanced glycosylation end products (AGE-R) may also play a role in senescent erythrocyte recognition $(9,10)$, the pattern of inhibition of recognition of aged neutrophils by polyanions did not support a role for the AGE-R. Therefore, the mechanism of $M \phi$ recognition of aged neutrophils does not resemble mechanisms proposed for the recognition of senescent erythrocytes.

There are other mechanisms by which unwanted senescent cells may be removed. Nucleated cells of many types undergo programmed cell death or apoptosis, a process characterized by stereotyped morphological changes (including nuclear chromatin condensation and cytoplasmic vacuolation due to dilatation of the endoplasmic reticulum) and evidence of endonuclease activation, with a specific pattern of DNA frag-

Proportion of Md ingesting PMN (as 8 of control)

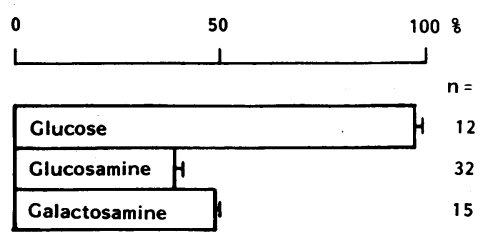

$10 \mathrm{mM}$ sugar in interaction medium

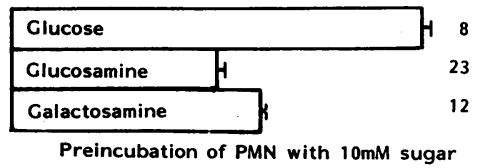

\begin{tabular}{|l|c|}
\hline Glucose & -1 \\
\hline Glucosamine & -1 \\
\hline Galactosamine & \\
\end{tabular}

Preincubation of $M \phi$ with $10 \mathrm{mM}$ sugar
Figure 6. Localization of the inhibitory effect of amino sugars. Preincubation at $4^{\circ} \mathrm{C}$ for 30 min of aged neutrophils, but not of $\mathbf{M} \phi$, with $10 \mathrm{mM}$ amino sugars, followed by washing, inhibited recognition to a degree comparable to that observed when these sugars were present in the interaction. In these experiments 47.1 $\pm 3.2 \%$ of $\mathrm{M} \phi$ recognized aged neutrophils tions. under control condi-

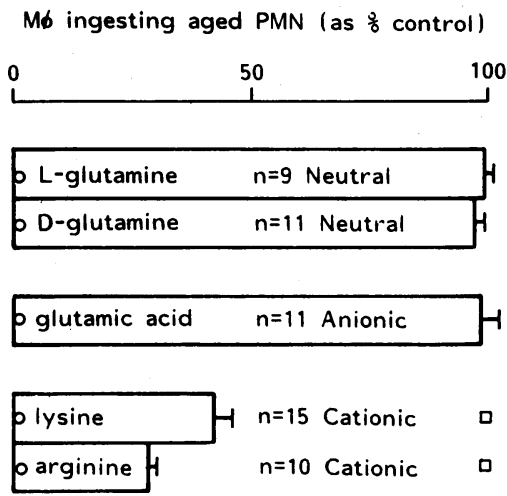

Figure 7. Effect of amino acids at $10 \mathrm{mM}$ and $\mathrm{pH}$ 7.4. The cationic, basic amino acids L-lysine and L-arginine inhibited macrophage interaction with aged neutrophils; basic and neutral amino acids had no effect. In these experiments $43.5 \pm 3.8 \%$ of $\mathbf{M} \phi$ recognized aged neutrophils under control conditions. Open squares, $\mathbf{M} \phi$ ingestion of EIgG (\%); open circles, \% PMN admitting trypan blue.

mentation $(4,5)$. Programmed cell death plays an important role in tissue kinetics since it leads to the removal of intact apoptotic cells by phagocytes; for example in embryological remodeling, or hormone-dependent thymic involution (4). In neutrophils isolated and aged by the methods described here, we have recently demonstrated a progressive increase in the proportion of cells with features typical of apoptosis (3), recognition by $\mathbf{M} \phi$ (assayed by the methods used here) being directly related to this process in the aging neutrophil (3). Unfortunately, since apoptosis is usually distributed broadly within tissues, there has been little study of the cell-surface interactions that lead to recognition of apoptotic cells as nonself. However, in a rosetting assay performed at $4^{\circ} \mathrm{C}$ it has been reported that the binding of apoptotic mouse thymocytes to isologous $\mathrm{M} \phi$ could be inhibited by 10 or $20 \mathrm{mM} N$-acetylglucosamine, $N$-acetylgalactosamine, and galactose (11). Preincubation experiments localized this effect to the $\mathrm{M} \phi$ surface, and it was suggested that a $M \phi$ lectin-like receptor might recognize putative changes in the surface carbohydrates of the apoptotic thymocyte, and that a similar process might apply to the recognition of other apoptotic cells. However, when these sugars were included at concentrations up to $50 \mathrm{mM}$ in the assay of phagocytosis of aged neutrophils at $37^{\circ} \mathrm{C}$ no evidence was obtained in support of a role for such a lectin. So far as these assays are comparable, and possible species differences should be noted, it did not appear likely that a human analogue of this putative structure played an important role in $\mathbf{M} \phi$ uptake of

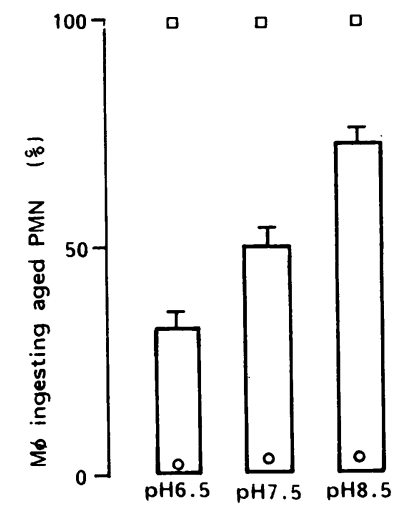

Figure 8. Effect of $\mathrm{pH}$ on the interaction of aged neutrophils with $\mathbf{M} \phi . \mathbf{M} \phi$ recognition of aged neutrophils (open bars) and EIgG (open squares) is expressed as absolute proportion of $\mathbf{M} \phi$ recognizing aged neutrophils. Open circles, $\%$ PMN admitting trypan blue; $n=24$. 
Proportion Mø ingesting PMN

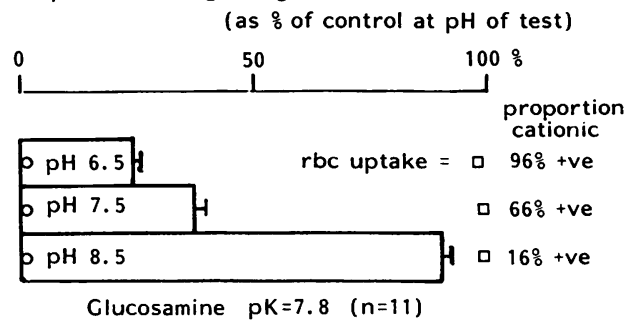

\begin{tabular}{|c|c|}
\hline ppH 6.5 & ․ $30 \%+v e$ \\
\hline $\mathrm{ppH} 7.5$ & $4 \%+v e$ \\
\hline $\mathrm{ppH} 8.5$ & $41 \%+v e$ \\
\hline
\end{tabular}

$\frac{p p H 7.5}{\text { L-lysine pKr=8.9 } 8.5}$

$96 \%+v e$

ㄱ $71 \%+v e$

Figure 9. Dependence of the inhibitory effect of $10 \mathrm{mM}$ amino sugars and amino acids on charge. The $\mathrm{pH}$ of the interaction influenced the degree of inhibition relative to the control at the same $\mathrm{pH}$, suggesting a relationship with the proportion of glucosamine or histidine calculated (see Methods) to be bearing a cationic charge (right column). The reduction of the inhibitory effect of these molecules at higher $\mathrm{pH}$ was not observed with L-lysine. In these experiments, at $\mathrm{pH} 7.546 .0 \pm 3.6 \%$ of $\mathrm{M} \phi$ recognized aged PMN under control conditions, at $\mathrm{pH} 0.5$ this figure was $32.2 \pm 3.4 \%$, and at $\mathrm{pH}$ $8.5,68.4 \pm 2.7 \%$. Open squares, $\mathrm{M} \phi$ uptake $\mathrm{EIgG}$; open circles, $\%$ PMN trypan blue positive.

aged neutrophils, particularly as inhibitors of human $\mathrm{M} \phi$ lectin-like receptors with similar sugar specificities (the mannosyl-fucosyl and asialoglycoprotein/galactosyl receptors) had no effect on the interaction. Indeed, the apparent localization of the inhibitory effect of amino sugars to the neutrophil surface and inhibition by amino acids are not consistent with the generally accepted concepts of a lectin-like molecule (27).

In this study it was observed that amino sugars and basic amino acids inhibited $M \phi$ recognition of aged neutrophils but not of opsonized erythrocytes, indicating that this effect was not due to a nonspecific impairment of $\mathbf{M} \phi$ phagocytic capability. Furthermore, the lack of effect of preincubation of these molecules with $\mathbf{M} \phi$ (followed by washing) indicated that inhibition was unlikely to reflect binding to $\mathbf{M} \phi$ receptors for aged neutrophils. However, molecules of both classes inhibited the subsequent recognition of aged neutrophils with which they were preincubated. As preincubations were done at $4^{\circ} \mathrm{C}$ it appeared likely that the inhibitory effect of both amino sugars and basic amino acids was directly on the aged neutrophil's surface, rather than on the intracellular metabolic processes leading to expression of aged neutrophil surface structures recognized by $\mathbf{M} \phi$. Indeed, the inhibitory effect of molecules of both classes at different $\mathrm{pHs}$ indicated that this effect was dependent on bearing a cationic charge in each case, a further indication that amino sugars and basic amino acids were influencing the interaction by similar mechanisms.

The possible nature of the inhibitory effect of amino sugars and basic amino acids was suggested by consideration of the other findings of this study. Although the pattern of inhibition of $\mathrm{M} \phi$ recognition of aged neutrophils was different from that of the AGE-R, inhibition by heparin suggested that $M \phi$ receptors for anionic ligands might be involved in the interaction. However, anionic competitive inhibitors of a number of wellcharacterized $\mathbf{M} \phi$ receptors for anionic ligands (the sheep erythrocyte, mannose-6-phosphate, and scavenger receptors) failed to inhibit the interaction. Nevertheless, the possible importance of anionic charge of aged neutrophil moieties recognized by $\mathbf{M} \phi$ receptors for these cells was highlighted by the direct effects of $\mathrm{pH}$ on the interaction; conditions in which anionic charge on aged neutrophil surface structures might be expected to increase (i.e., raising $\mathrm{pH}$ ) were associated with potentiation of the interaction. Furthermore, the localization of the inhibitory effect of amino sugars and basic amino acids

Table II. Lack of Effect of Inhibitors of Known Macrophage Receptors on Aged Neutrophil Recognition

\begin{tabular}{|c|c|c|c|}
\hline Receptor & $\begin{array}{c}\text { Inhibitor } \\
\text { (maximum concentration) }\end{array}$ & $\begin{array}{c}\text { Aged PMN } \\
\text { (\% of control } \pm \text { SE, } n=12 \text { ) }\end{array}$ & Reference \\
\hline \multirow[t]{4}{*}{ Mannose-6-phosphate } & Mannose-6-phosphate (10 mM) & $101.8 \pm 2$ & 30,31 \\
\hline & Fructose-6-phosphate (20 mM) & $100.8 \pm 2.4$ & \\
\hline & Fructose-1-phosphate (20 mM) & $98.9 \pm 3.7$ & \\
\hline & $\alpha$-Mannosidase $(200 \mathrm{mg} / \mathrm{ml})$ & $102.8 \pm 3.1$ & \\
\hline \multirow[t]{2}{*}{ Sheep erythrocyte } & Ganglioside GDla (100 mg/ml) & $98.9 \pm 1.9$ & 32,33 \\
\hline & Neuraminyl-lactose (20 $\mathrm{mM})$ & $101.0 \pm 2.3$ & \\
\hline \multirow[t]{2}{*}{ Scavenger } & Dextran sulfate $(1 \mathrm{mg} / \mathrm{ml})$ & $107.5 \pm 3.1^{*}$ & $34-36$ \\
\hline & Fucoidin $(1 \mathrm{mg} / \mathrm{ml})$ & $102.2 \pm 2.6^{*}$ & \\
\hline \multirow[t]{2}{*}{ Mannosyl-fucosyl } & Ribonuclease B (1 mg/ml) & $101.6 \pm 0.9$ & $18,37,38$ \\
\hline & Mannan $(1 \mathrm{mg} / \mathrm{ml})$ & $100.2 \pm 2.6$ & \\
\hline \multirow{3}{*}{$\begin{array}{l}\text { Asialoglycoprotein } \\
\text { (Galactosyl-R) }\end{array}$} & $N$-Acetylgalactosamine (100 mM) & $104.2 \pm 4.3$ & $39-41$ \\
\hline & Galactose $(50 \mathrm{mM})$ & $99.0 \pm 1.2$ & \\
\hline & L-Fucose (50 mM) & $98.8 \pm 2.7$ & \\
\hline
\end{tabular}

In these experiments the mean proportion of $\mathrm{M} \phi$ recognizing aged PMN under control conditions was $49.1 \pm 4.5 \%$, $95 \%$ of observations falling within $9.5 \%$ of the mean of control $(100 \%)$. Glucosamine $(10 \mathrm{mM})$ in the same series of experiments inhibited aged neutrophil recognition to $40.1 \pm 2.2 \%$ of control. ${ }^{*}$ See also Fig. 2 . 
to the aged neutrophil surface also supports the importance of anionic charge on aged neutrophil surface moieties; the association of cationic molecules with the neutrophil surface would be expected to disturb anionic charges and thus inhibit the interaction if the anionic charge on neutrophil structures involved in the interaction were an important component of the recognition mechanism. Similar effects might account for the inhibitory effects of raised hydrogen ion concentrations. However, because only one of a number of anionic molecules tested inhibited the interaction it appeared very unlikely that anionic charge per se represented the sole recognition signal to the $\mathbf{M} \phi$, in which case inhibition would have been expected with all anionic molecules. Therefore, rather than suggesting that the surface charge density of the whole neutrophil represents the signal to the $\mathbf{M} \phi$, these data imply that the anionic charge on specific aged neutrophil sites is an important component of the recognition mechanism.

To conclude, it would appear that $\mathbf{M} \phi$ recognition of aged, apoptotic neutrophils depends on a novel recognition mechanism, different from those proposed for recognition of aged erythrocytes and apoptotic thymocytes, and not involving a number of $\mathrm{M} \phi$ receptors for which there were grounds to suggest a possible role. To understand the inhibitory effects of amino sugars, basic amino acids, and heparin on the interaction, and the basis for modulation by $\mathrm{pH}$, the structures involved in this recognition process need to be defined. Our findings show that the recognition mechanism has properties that may be summarized as "charge sensitive," which have important implications for the fate of the neutrophil at inflamed sites in vivo. Since perturbations of interstitial $\mathrm{pH}$ and release of charged molecules into the extracellular environment are well documented in inflammation $(26,28,29)$, our data suggest that these factors could influence the degree to which senescent inflammatory neutrophils are removed in situ by phagocytosis while still intact. It can be speculated that failure of this process, perhaps related to a low tissue fluid $\mathrm{pH}$ or a particular mix of charged molecules, might lead to greater release of potentially histotoxic contents from disintegrating neutrophils and therefore to exacerbation and persistence of tissue injury.

\section{Acknowledgments}

The authors wish to thank Dr. Gordon Ross for his detailed advice and the gifts of complement-coated red cells and MAbs (for which we are also indebted to Dr. Nancy Hogg and their colleagues), Dr. Deidre Grennan for the gift of rabbit IgG, Dr. David Riches for advice, Dr. Mark Walport and Dr. Joel David for synovial fluid, Alison Lee and Sarah Hall for expert technical assistance, and Brenda Johnson for preparing the manuscript.

This work was supported by the Medical Research Council of Great Britain, and National Institutes of Health grant HL-27353. Dr. Savill is in receipt of a Medical Research Council (MRC) Training Fellowship and Dr. Haslett is a MRC Senior Clinical Fellow.

\section{References}

1. Metchnikoff, E. 1891. Lectures on the Comparative Pathology of Inflammation. Lecture VII. F. A. Starling and E. H. Starling, translators. Dover Publications, Inc., New York. 106.

2. Newman, S. L., J. E. Henson, and P. M. Henson. 1982. Phagocytosis of senescent neutrophils by human monocyte-derived macro- phages and rabbit inflammatory macrophages. J. Exp. Med. 156:430442.

3. Savill, J. S., A. H. Wyllie, J. E. Henson, M. J. Walport, P. M. Henson, and C. Haslett. 1989. Macrophage phagocytosis of aging neutrophils in inflammation. Programmed cell death in the neutrophil leads to its recognition by macrophages. J. Clin. Invest. 83:865-875.

4. Wyllie, A. H., J. F. R. Kerr, and A. R. Currie. 1980. Cell death: the significance of apoptosis. Int. Rev. Cytol. 68:251-306.

5. Wyllie, A. H. Glucocorticoid-induced thymocyte apoptosis is associated with endogenous endonuclease activation. Nature (Lond.) 284:555-556.

6. Kay, M. M. B. 1975. Mechanism of removal of senescent cells by human macrophages in situ. Proc. Natl. Acad. Sci. USA. 72:35213525.

7. Kay, M. M. B. 1978. Role of physiologic autoantibody in the removal of senescent human red cells. J. Supramol. Struct. 9:555-567.

8. Gattegno, L., and L. Saffar. 1987. Involvement of monocytemacrophage complement receptors in the recognition of senescent human red blood cells. Presented at XIIth International Complement Workshop, Chamonix, France. (Abstr.)

9. Vlassara, H., M. Brownlee, and A. Cerami. 1986. Novel macrophage receptor for glucose-modified proteins is distinct from previously described scavenger receptors. J. Exp. Med. 164:1301-1309.

10. Vlassara, H., J. Valinsky, M. Brownlee, C. Cerami, S. Nishimoto, and A. Cerami. 1987. Advanced glycosylation end-products on erythrocyte cell surface induce receptor-mediated phagocytosis by macrophages. J. Exp. Med. 166:539-549.

11. Duvall, E., A. H. Wyllie, and R. G. Morris. 1985. Macrophage recognition of cells undergoing programmed cell death (apoptosis). Immunology. 56:351-358.

12. Haslett, C., L. A. Guthrie, M. M. Kopaniak, R. B. Johnston, and P. M. Henson. 1985. Modulation of multiple neutrophil functions by preparative methods or trace concentrations of bacterial lipopolysaccharide. Am. J. Pathol. 119:101-110.

13. Henson, P. M., B. Zanolari, N. A. Schwartzman, and S. R. Hong. 1978. Intracellular control of human neutrophil secretion. I. C5a-induced stimulus-specific desensitisation and the effects of cytochalasin B. J. Immunol. 121:851-855.

14. Ross, G. D., J. A. Cain, and P. J. Lachmann. 1985. Membrane complement receptor type three (CR3) has lectin-like properties analagous to bovine conglutinin and functions as a receptor for zymosan and rabbit erythrocytes as well as a receptor for iC3b. J. Immunol. 134:3307-3315.

15. Myons, B. L., J. G. Dalzell, N. H. Hogg, and G. D. Ross. 1988. Neutrophil and monocyte cell surface p150,95 has iC3b-receptor (CR4) activity resembling CR3. J. Clin. Invest. 82:640-651.

16. Dalzell, J. G., G. D. Ross, and S. Becker. 1988. Expression and ligand-binding activity of complement receptors on alveolar macrophages. FASEB (Fed. Am. Soc. Exp. Biol.) J. 2:A685. (Abstr.)

17. Riches, D. W. H., and D. R. Stanworth. 1982. Evidence for a mechanism for the initiation of acid hydrolase secretion by macrophages that is functionally independent of alternate pathway complement interaction. Biochem. J. 202:639-645.

18. Ezekowitz, R. A. B., and P. D. Stahl. 1988. The structure and function of vertebrate mannose lectin-like proteins. J. Cell Sci. Suppl. 9:121-133.

19. Brewer, D. B. 1964. Electron-microscope phagocytosis of neutrophil polymorphonuclear leukocytes by macrophages. J. Pathol. 88:307-309.

20. Pekin, T., T. I. Malinin, and N. J. Zwaifler. 1967. Unusual synovial fluid findings in Reiter's syndrome. Ann. Int. Med. 66:677684.

21. Nichols, B. A., and D. F. Bainton. 1975. Ultrastructure and cytochemistry of mononuclear phagocytes. In Mononuclear Phagocytes in Immunity, Infection and Pathology. R. van Furth, editor. Blackwell Scientific Publications Ltd., London. 39.

22. Spriggs, R. S., M. M. Boddington, and A. G. Mowat. 1978. Joint fluid cytology in Reiter's disease. Ann. Rheum. Dis. 37:557-560. 
23. Sanui, H., S.-i. Yoshida, K. Nomoto, R. Ohhara, and Y. Adachi. 1982. Peritoneal macrophages which phagocytose autologous polymorphonuclear leucocytes in guinea-pigs. I. Induction by irritants and microorganisms and inhibition by colchicine. Br. J. Exp. Pathol. 63:278-285.

24. Chapes, S. K., and S. Haskill. 1983. Evidence for granulocytemediated macrophage activation after $C$. parvum Immunization. Cell. Immunol. 75:367-377.

25. Cochrane, C. G. 1968. Immunologic tissue injury mediated by neutrophilic leukocytes. $A d v$. Immunol. 9:97-162.

26. Henson, P. M., and R. B. Johnston. 1987. Tissue injury in inflammation. Oxidants, proteinases, and cationic proteins. J. Clin Invest. 79:669-674.

27. Brandley, B. K., and R. L. Schnaar. 1986. Cell-surface carbohydrates and cellular injury. Science (Wash. DC). 123:527-534.

28. Menken, V. 1956. Biology of inflammation: chemical mediators and cellular injury. Science (Wash. DC). 123:527-534.

29. Hunt, T. K., M. J. Banda, and I. A. Silver. 1985. Cell interactions in post-traumatic fibrosis: fibrosis (Ciba Found. Symp.) 114:127-149.

30. Shepherd, V. L., H. H. Freeze, A. L. Miller, and P. D. Stahl. 1984. Identification of mannose-6-phosphate receptors in rabbit alveolar macrophages. J. Biol. Chem. 259:2257-2261.

31. Hoflack, B., and S. Kornfeld. 1985. Lysosomal enzyme binding to mouse P388D, macrophage membranes lacking the $215-\mathrm{kDa}$ mannose-6-phosphate receptor: evidence for the existence of a second mannose-6-phosphate receptor. Proc. Natl. Acad. Sci. USA. 82:44284432.

32. Crocker, P. R., and S. Gordon. 1986. Properties and distribution of a lectin-like hemagglutinin differentially expressed by murine stromal tissue macrophages. J. Exp. Med. 164:1862-1875.

33. Crocker, P. R., L. Morris, and S. Gordon. 1988. Novel cell surface adhesion receptors involved in interactions between stromal macrophages and hematopoietic cells. J. Cell Sci. Suppl. 9:185-206.

34. Goldstein, J. L., Y. K. Ho, S. K. Basu, and M. S. Brown. 1979. Binding site on macrophages that mediates uptake and degradation of acetylated low density lipoprotein, producing massive cholesterol deposition. Proc. Natl. Acad. Sci. USA. 76:333-337.

35. Brown, M. S., S. K. Basu, J. R. Falck, Y. K. Ho, and J. L. Goldstein. 1980. The scavenger cell pathway for lipoprotein degradation: specificity of the binding site that mediates the uptake of negatively-charged LDL by macrophages. J. Supramol. Struct. 13:67-81.

36. Haberland, M. E., R. R. Rasmussen, C. L. Olch, and A. L. Fogelman. 1986. Two distinct receptors account for recognition of maleyl-albumin in human monocytes during differentiation in vitro. $J$. Clin. Invest. 77:681-689.

37. Stahl, P., and S. Gordon. 1982. Expression of a mannosyl-fucosyl receptor for endocytosis on cultured primary macrophages and their hybrids. J. Cell Biol. 93:49-56.

38. Blackwell, J. M., R. A. B. Ezekowitz, M. B. Roberts, J. Y. Channon, R. B. Sim, and S. Gordon. 1985. Macrophage complement and lectin-like receptors bind Leishmania in the absence of serum. $J$. Exp. Med. 162:324-331.

39. Schlepper-Schafer, J., V. Kolb-Bachofen, and H. Kolb. 1980. Analysis of lectin-dependent recognition of desialylated erythrocytes by Kuppfer cells. Biochem. J. 186:827-831.

40. Kolb-Bachofen, V., J. Schlepper-Schafer, and W. Vogell. 1982. Electron microscopic evidence for an asialoglycoprotein on Kuppfer cells: localisation of lectin-mediated endocytosis. Cell. 29:859-862.

41. Roos, P. H., H.-J. Hartman, J. Schlepper-Schafer, H. Kolb, and V. Kolb-Bachofen. 1985. Calactose-specific receptors on liver cells. II. Characterization of the purified receptor from macrophages reveals no structural relationship to the hepatocyte receptor. Biochim. Biophys. Acta. 847:115-121. 